\title{
Implementation of a rapid antibody based method to detect Pichia sp. and Hanseniaspora uvarum during the whole winemaking process
}

\author{
F. $\operatorname{Rex}^{1, a}$ and M. Scharfenberger-Schmeer ${ }^{1,2,3}$ \\ ${ }^{1}$ Institute for Viticulture and Oenology, Dienstleistungszentrum Ländlicher Raum (DLR) Rheinpfalz, Breitenweg 71, 67435 \\ Neustadt, Germany \\ ${ }^{2}$ Hochschule Kaiserslautern, Schönstraße 11, 67663 Kaiserslautern \\ ${ }^{3}$ Weincampus Neustadt, Breitenweg 71, 67435 Neustadt, Germany
}

\begin{abstract}
Off-flavors produced by spoilage microorganisms should be avoided during fermentation. In case of spontaneous fermentation, difficult storage conditions or long-lasting transport, it is necessary to detect spoilage microorganisms before population sizes achieve the critical level to produce perceivable off-flavors. Additionally, a knowledge of the composition of microorganisms allows winemakers to reduce treatments such as $\mathrm{SO}_{2}$ addition. For this reason a rapid antibody-based analytic method was developed providing the winemaker with the information about beneficial and harmful microorganisms without the need of a laboratory equipment and lengthy wait periods. Antibodies for the detection of the genera Pichia and Hanseniaspora were generated. For the evaluation of the new antibody based method an ELISA test system has been used showing a high sensitivity beginning with $10^{3}$ cells $/ \mathrm{ml}$ and a specificity to the spoilage yeast. All antibodies are designed to detect microorganisms worldwide. The following industrial production of the rapid method will deliver a tool for winemakers and distributive traders to get a view inside the ongoing fermentation within twenty minutes by using a diagnostic dipstick. With the help of the antibody-based detection method the quality of the wine can be increased and economic risks can be minimized at the same time.
\end{abstract}

\section{Introduction}

Different yeast species can be found during fermentation. Generally the desired one is Saccharomyces cerevisiae. But there are also spoilage yeasts which can lead to off-flavors. One of these is the genus Pichia. Some of the species can form acetaldehyde, acetic acid and ethyl acetate in the presence of oxygen, which have a negative effect on the flavor of the wine. In addition, they form an unappetizing film on the surface of the wine [1]. These yeasts on the surface of the wine also have the negative effect that they enable the development of acetic acid bacteria additionally causing damage to the wine. The specie Hanseniaspora uvarum (anamorph: Kloeckera apiculata) is lemon-like shaped and can form high amounts of volatile acid, ethyl acetate or hydrogen sulphide, depending on the strain [2].

The problem of contaminations with spoilage yeasts increased during the last years. As a result of climate change, rising $\mathrm{pH}$-values in the must are observed in many wine growing regions and are expected to increase over the next years. This promotes the conditions for spoilage microorganisms and reduces the microbicidal effect of $\mathrm{SO}_{2}$ at the same time. In addition, the first $\mathrm{SO}_{2}$ tolerant Brettanomyces bruxellensis strains are observed [3] and other species might follow these tendencies. Therefore, the wine industry must focus on alternatives to reduce the need of $\mathrm{SO}_{2}$ for wine stabilization. First of all, it must be

a e-mail: friederike.rex@dlr.rlp.de the goal to avoid infections with spoilage yeasts during the beginning of the fermentation. To control if there is an infection the methods for the analysis of yeast and bacteria are cost and time intensive due to the required laboratory and technical expertise. In addition, the analysis result is only available after days and too late to conduct countermeasures. Except of using a microscope all tests like the determination of CFU [4] or methods as qPCR or sequencing are not delivering quick results [5]. However, in case of contamination there is no time to wait for results. The winemaker has to apply appropriate treatments such as $\mathrm{SO}_{2}$ addition, filtration or pasteurization before offflavors become obvious. At the same time, the microbial stability after filtration and bottling as well as during global transport and trade can be significantly increased with a knowledge of the microflora. This reduces costs and increases the quality of the wine and the success of the product. Therefore there is a great need for a simple and inexpensive method for the rapid detection of microorganisms during all stages of production.

Immunological rapid tests in the form of a dipstick test fulfill these requirements. These tests can be applied directly to must or wine by simply immersing the test strip without any special technical effort or fundamental microbiological skills needed. The basis for the test kit is the development of specific antibodies. The development of antibodies was already published for the detection of Brettanomyces [6] and showed good results in direct wine samples. But the mentioned work did not result 
in a test system. In our project the antibodies for the harmful yeasts Pichia sp. and Hanseniaspora uvarum, were developed and tested for their specificity, sensitivity and applicability in wine by using an ELISA (enzymelinked immunosorbent assay) method.

\section{Material and methods}

\subsection{Yeasts and cultivation}

The yeasts used for the experiments are part of the collection of microorganisms at the DLR Rheinpfalz and isolated from must and wine samples or received from the Leibniz institute DSMZ (German collection of microorganisms and cell cultures Braunschweig, Germany) and from the collection of the Westerdijk fungal biodiversity institute, Utrecht, the Netherlands. Yeast extract-peptone-dextrose (YPD) media was autoclaved for the cultivation. Cells were counted with a Neubauer improved counting chamber and adjusted to $10^{6}$ cells $/ \mathrm{ml}$ if not mentioned otherwise.

\subsection{Immunization}

The immunization for the antibody against Pichia sp. was developed by BioGenes $\mathrm{GmbH}$, Berlin, Germany and the antibody against $H$. uvarum by Immundiagnostik $\mathrm{GmbH}$, Bensheim, Germany according to their own protocol. After the immunization, the titer achieved was determined and the antiserum recovered. The antiserum obtained was chromatographed on a protein-A column, whereby the pure antibody preparation was recovered. This was checked for suitability, adjusted to the required concentration and used for the test kit.

\subsection{Elisa}

Yeast cells in YPD-Medium or wine samples $(100 \mu \mathrm{l})$ were centrifuged $(2 \mathrm{~min}$ at $1000 \mathrm{rpm})$ and the pellet was resuspended in $500 \mu \mathrm{l}$ PBS solution (pH 10.2). $100 \mu \mathrm{l}$ were coated in a polystyrene plate (BRANDplates ${ }^{\circledR}-$ immunoGrade ${ }^{\mathrm{TM}}$, Brand $\mathrm{GmbH}$ und $\mathrm{Co} \mathrm{KG}$, Wertheim, Germany) overnight at $4{ }^{\circ} \mathrm{C}$. The plate was blocked with a 3\% bovine serum albumin (BSA, Albumin Fraction $\mathrm{V}$ pH 7.0, AppliChem GmbH, Darmstadt, Germany) in PBS solution for one hour at RT. $100 \mu$ of the yeastspecific primary antibody (diluted 1:500 with PBS pH 7.2-7.4) were added. After incubation for $1.5 \mathrm{~h}$ at RT $95 \mu \mathrm{L}$ alcaline phosphatase conjugate Goat-anti-rabbit Antibody (diluted 1:2000 with PBS 7.2-7.4, Jackson Immuno Research, Ely, UK) was added. The plate was incubated for $2 \mathrm{~h}$ at RT. The signal was developed by adding $95 \mu \mathrm{L}$ substrate $(0.2 \mathrm{M}$ Tris $\mathrm{pH} 8.8+5 \mathrm{mM}$ $\mathrm{MgCl}_{2}+1 \mathrm{mg} / \mathrm{mL}$ para-nitrophenylphosphat, Carl Roth $\mathrm{GmbH}$, Karlsruhe, Germany; Tris, AppliChem GmbH, Darmstadt, Germany) and then measured using an ELISA absorbance Ao microplate reader (Azure Biosystems, inc., Dublin, California, USA) at $405 \mathrm{~nm}$.

\section{Results}

\subsection{The antibody against the genus Pichia}

The antibody for the detection of Pichia sp. is a polyclonal antibody and it is sensitive for the detection

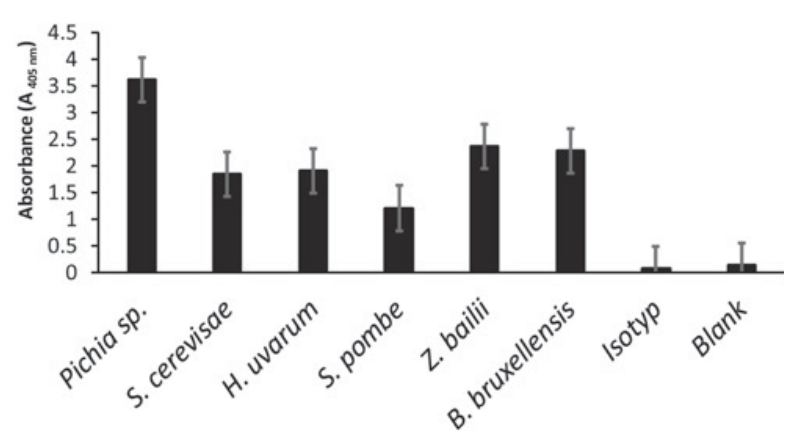

Figure 1. ELISA results of the crossreactions of the antibody against Pichia with other wine-related yeasts.

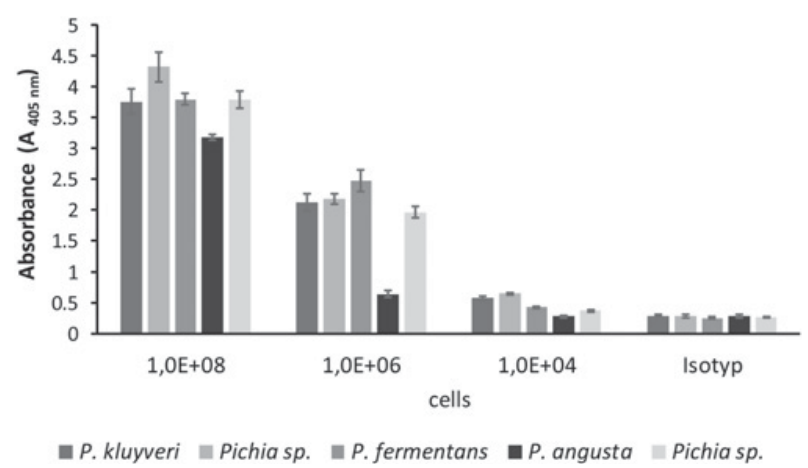

Figure 2. Results of the ELISA using different Pichia strains and species. The antibody can be used for detecting the whole genus Pichia and is able to quantify cell numbers from $10^{4}-10^{8}$ cells $/ \mathrm{ml}$.

of cells with an LOQ of $10^{3}$ cells/ml (data not shown). It also reacts with some other wine associated yeasts S. cerevisiae, H. uvarum, Schizosaccharomyces pombe, Zygosaccharomyces bailii and B. bruxellensis but the absorbance level using an ELISA is the most intensive whereby the signal for Pichia can be differentiated (Fig. 1). The antibody binds to all species of Pichia: two Pichia sp. strains, Pichia angusta, Pichia fermentans and Pichia klyuveri (Fig. 2). The antibody can be used during the whole vinification process and the increasing level of alcohol does not influence the results (data not shown).

\subsection{The antibody against Hanseniaspora uvarum}

The polyclonal antibody for the detection of H. uvarum is specific. There are negligible cross reactions with other wine associated yeasts (Fig. 3). It can quantify cell numbers beginning with $10^{3}$ cells $/ \mathrm{ml}$. It binds to H. uvarum strains isolated in all parts of the world (Fig. 4).

\section{Discussion}

The alcoholic fermentation can be influenced negatively by spoilage yeasts as Pichia sp. and H. uvarum and a quick method to detect these organisms will be a helpful tool to increase wine quality. To achieve this goal the development of antibodies against these species was conducted and a protocol for their application in an ELISA was established.

The presented data showed that two antibodies are ready to be integrated in a dipstick test system. The 


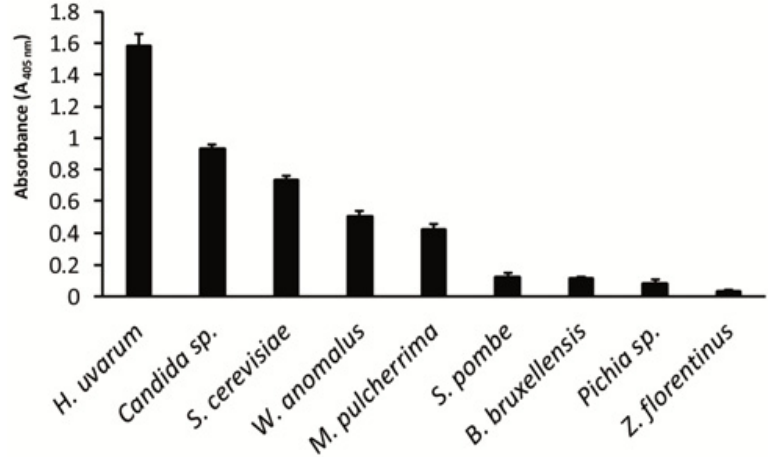

Figure 3. ELISA results of the test of cross reactions. The level of absorbance shows a signal for H. uvarum.

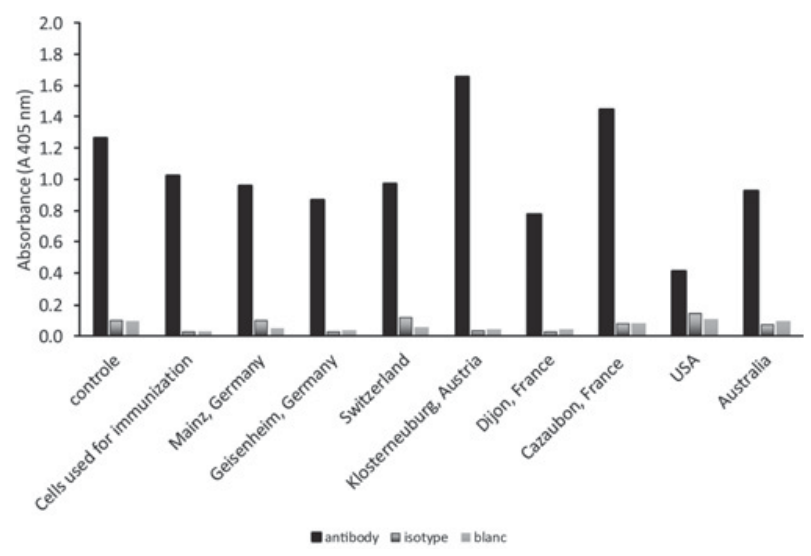

Figure 4. ELISA results using $H$. uvarum strains isolated in different parts of the world, in Germany, in Switzerland, in Austria, in France, in Australia and in the USA. The level of absorbance of these strains is as high as the controls.

antibody against the genera Pichia allows the detection of cells in pure cultures with a LOQ of $10^{3}$ cells $/ \mathrm{ml}$ with a maximum of $10^{8}$ cells $/ \mathrm{ml}$. The LOQ is low enough to detect this spoilage yeast at a cell density before any off-flavor can occur. The advantage of the antibody is its ability to detect all tested species of the genera Pichia (Fig. 2), which reduces the costs for the testkit "Pichia". Another advantage of this antibody is its capacity to bind to P. kluyveri which is a commercially available non-Saccharomyces strain (Frootzen, Chr. Hansen, Hoersholm, Danmark), producing fruity volatile thiols during fermentation [13]. The cell number must be 106 cells $/ \mathrm{ml}$ after inoculation which could be checked with a test strip. The remaining disadvantage is the cross-reaction with other yeast species (Fig. 1), therefore the implication in a dipstick method is still in progress.

The antibody against $H$. uvarum is specific for the target organism and shows negligible cross-reactivity. The LOQ is $10^{4}$ cells $/ \mathrm{ml}$. The application on a universal test strip was successful and detection of $H$. uvarum is possible within $20 \mathrm{~min}$. It could be shown, that the antibody is highly sensitive against any $H$. uvarum strain, independent from the origine (Fig. 4). This result is very important, showing that the application of the dipstick method is not limited to a specific winegrowing region. Thus, the rapid test is internationally applicable.
With this study two antibodies are established. Further steps have to be done to realize the lateral flow dipstick test and an application software (Cooperation with Immundiagnostik AG, Bensheim, Germany) to assist the quantification. The immunization and work with polyclonal whole IgG-molecules turned out not to be the most effective way to develop a rapid method. This can be caused by the changes of cell wall structures in subject to the cell cycle and changes of the environmental conditions [7-9]. Immunization was also conducted for numerous other spoilage yeasts as $S$. pombe, B. bruxellensis and as a positive detection $S$. cerevisiae, but the resulting antibodies offered too many cross-reactions (data not shown), which can be caused by similar yeast cell wall structures [10-12].

To overcome this problem, phage display technology to select a monoclonal specific scFV (single chain variable fragment) is applied starting with an antibody fragment against B. bruxellensis. The selected scFv can be produced in bacteria in unlimited amounts. In addition to the spoilage yeasts mentioned, bacteria can also cause problems during fermentation and even in the wine. To establish a detection system antibodies against the genera Gluconobacter, Pediococcus, Lactobacillus and Acetobacter will be developed by phage display technology as well. The industrial production of several dipstick assays will provide winemakers and traders a tool to investigate and follow the microbial status for all abovementioned species or genera. For the worldwide trade the developed method is an advantage as the microbial status (such as contamination with Brettanomcyes or bacteria) of the wine can be recorded within a few minutes before and after its shipment. Thus, it can be ensured that only non-contaminated wine will be shipped and the spoiling of large quantities is prevented. Since the method is easy to use, fast and inexpensive, it can be used at all stages of the production. With the help of the antibody based detection method, the quality of the wines can be increased and the economic risks can be minimized.

The IGF Project (AIF 18676 N) of the FEI is supported via AiF within the program for promoting the Industrial Collective Research (IGF) of the German Ministry of Economics and Energy (BMWi)

\section{References}

[1] V. Loureiro, M. Malfeito-Ferreira, Int. J. Food Microbiol. 86, 23 (2003)

[2] V. Martin, M.J. Valera, K. Medina, E. Boido, F. Carrau, Fermentation 4, 76 (2018)

[3] M. Avramova, A. Vallet-Courbin, J. Maupeu, I. Masneuf-Pomaréde, W. Albertin, Front. Microbiol. 9, 1260 (2018)

[4] D. Schuller, M. Corte-Real, C. Leao, J. Food Protect. 63, 1570 (2000)

[5] H. Rawsthorne, T.G. Phister, Int. J. Food Microbiol. 112, 1 (2006)

[6] A.H. Kuniyuki, C. Rous, J.L. Sanderson, Am. J. Enol. Vitic. 35, 143 (1986)

[7] N. Anfang, M. Brajkovich, M.R. Goddard, Aust. J. Grape Wine R. 15, 1 (2009) 
[8] F.M. Klis, P. Mol, K. Hellingwerf, S. Brul FEMS Microbiol Rev. 26, 239 (2002)

[9] F.M. Klis, A. Boorsma, P.W.J. De Groot, Yeast 23, 185 (2006)

[10] B. Aguilar-Uscanga, J.M. Francois, Lett. Appl. Microbiol. 37, 268 (2003)
[11] R. Kollár, E. Petráková, G. Ashwell, P.W. Robbins, E. Cabib, J. Biol. Chem. 270, 1170 (1995)

[12] P.N. Lipke, R. Ovalle, J. Bacteriol. 180, 3735 (1998)

[13] M.P. Schreuder, A.T. Mooren, H.Y. Toschka, C.T. Verrips, F.M. Klis, Trends Biotech. 14, 115 (1996) 